Para enlazar con este artículo / To link to this article:

http://dx.doi.org/10.6035/MonTI.2019.11.7

Para citar este artículo / To cite this article:

Cid-Leal, Pilar; María-Carmen Espín-García \& Marisa Presas. (2019) “Traducción automática y posedición: Perfiles y competencias en los programas de formación de traductores.” En: Tolosa Igualada, Miguel \& Álvaro Echeverri (eds.) 2019. Porque algo tiene que cambiar. La formación de traductores e intérpretes: Presente $\mathcal{E}$ futuro / Because something should change: Present \& Future Training of Translators and Interpreters. MonTI 11 , pp. 187-214.

\title{
TRADUCCIÓN AUTOMÁTICA Y POSEDICIÓN: PERFILES Y COMPETENCIAS EN LOS PROGRAMAS DE FORMACIÓN DE TRADUCTORES ${ }^{1}$
}

\author{
Pilar Cid-Leal \\ Pilar.Cid@uab.cat \\ Universitat Autònoma de Barcelona \\ María-Carmen Espín-García \\ Carmen.Espin@uab.cat \\ Universitat Autònoma de Barcelona \\ Marisa Presas \\ Marisa.Presas@uab.cat \\ Universitat Autònoma de Barcelona
}

\section{Resumen}

Los estudios sobre el mercado profesional de la traducción muestran que cada vez más los empleadores demandan que los traductores dominen las herramientas relacionadas con traducción automática. Esto obliga a los centros de formación de traductores a preguntarse cuáles son los nuevos perfiles profesionales y cuáles son las nuevas competencias que deberían adquirir los estudiantes. En este artículo reflexionamos sobre estos aspectos a partir de una revisión bibliográfica y del análisis de las páginas web de grados y másteres de traducción impartidos en las universidades españolas en el curso 2017-2018. Este análisis se ha centrado en la presencia de la traducción automática (TA) y posedición (PE) en los enunciados de perfiles profesionales, competencias y contenidos. Nuestros resultados indican que el aprendizaje de esta tecnología ocupa aún un lugar marginal en los programas de formación de traductores. El artículo concluye con la propuesta de un marco general de perfiles y competencias que integra la TA y la PE.

1. El presente trabajo forma parte del proyecto ProjecTA-U, del grupo Tradumàtica (FFI2016-78612-R). 


\begin{abstract}
"Machine translation and post-editing: profiles and competences in translator training programmes"

Studies on the professional translation market show that employers increasingly demand translators who master tools related to machine translation. This fact compels translator training centers to consider what the new professional profiles are and what new competences students should acquire. In this article we reflect on these two aspects through a literature survey and an analysis of the web pages of undergraduate and master's degrees in translation taught at Spanish universities in academic year 2017-2018. Our analysis has focused on the presence of machine translation (MT) and post-editing (PE) in professional profiles, competencies and contents described in academic curricula. Our results indicate that the learning of this technology plays a marginal role in translator training programmes. The article concludes with a proposal of a general framework of competences which integrates both MT and PE.

Palabras clave: Traducción automática. Posedición. Formación de traductores. Perfil profesional. Competencias tecnológicas.

Keywords: Machine translation; Post-editing; Translator training; Professional profile; Technological competences.
\end{abstract}




\section{Introducción}

En los últimos decenios el desarrollo de las tecnologías ha cambiado la práctica de la traducción. La traducción automática (TA), entendida como la producción de textos traducidos de una lengua natural a otra con o sin intervención humana, representa el cambio más profundo para la función del traductor. La TA conlleva la aparición de nuevas tareas como la preedición y la posedición (PE). En el flujo de trabajo con TA la preedición consiste, a grandes rasgos, en modificar el contenido en lengua original antes de ser traducido de forma automática para facilitar el proceso y mejorar su resultado; de esta manera se reduce la carga de posedición. A su vez, la PE consiste en la edición y corrección del resultado de la traducción automática. De acuerdo con los requisitos de calidad definidos en el encargo la PE puede ser parcial o completa. En términos generales, la $\mathrm{PE}$ completa pretende obtener un producto comparable a la traducción humana, mientras que la PE parcial se dirige a obtener un texto que sea comprensible para su destinatario.

Debido a la importancia de la traducción en la comunicación internacional, empresas e instituciones públicas están invirtiendo una gran cantidad de recursos en el desarrollo y mejora de sistemas de TA. Esta tecnología está considerada como un sector estratégico por la Unión Europea, con 24 lenguas de trabajo. Varios informes (DePalma, Hegde, Pielmeier \& Stewart 2014; Varios autores -EUATC - 2017; Van der Meer \& Ruopp 2014, entre otros) destacan que la TA está considerada por empresas y profesionales como la herramienta del futuro. La previsión es que el uso de las herramientas de traducción en general y de traducción automática en particular experimentará una expansión debido al volumen creciente de material para traducir, por lo que también crecerá la demanda de traductores que puedan utilizar los sistemas de TA adecuadamente.

En el sector de los servicios lingüísticos, tanto en las empresas como entre los profesionales, está consolidado el uso de herramientas de traducción asistida por ordenador (TAO). Los traductores utilizan estas herramientas para tareas como la propia traducción, la gestión terminológica o la gestión de proyectos (Zaretskaya, Corpas Pastor \& Seghiri Domínguez 2015). En los últimos 
25 años las herramientas de TAO no han experimentado cambios esenciales en sus prestaciones o, en todo caso, las innovaciones han tenido escaso impacto en el proceso traductor (Sánchez-Gijón 2016: 154). En contraste, la implantación de un sistema de TA implica importantes cambios en la manera de trabajar: traducir ya no consiste en producir un texto en lengua meta a partir de un texto en lengua original, sino que consistiría en validar el producto de la TA. Aunque esta afirmación puede y debe ser matizada, este cambio explicaría por qué el crecimiento de la TA es, de acuerdo con algunos estudios, una de las mayores preocupaciones, sobre todo entre los profesionales. Según el trabajo llevado a cabo por el grupo Tradumàtica (Presas, Cid-Leal \& Torres-Hostench 2016), esta preocupación se debe en gran parte a la falta de formación específica de los traductores.

Las empresas de servicios lingüísticos y los profesionales pueden optar por trabajar con sistemas propios de TA o con sistemas de propósito general disponibles de manera gratuita en internet. En el primer caso, cabe distinguir entre sistemas desarrollados por la misma empresa o desarrollados por terceros por encargo. En este sentido, empresas como KantanMT o LetsMT permiten entrenar sistemas de TA propios 'en la nube' sin tener que instalar nada en la empresa. En este primer grupo, por tanto, los sistemas se adaptan a las necesidades específicas de cada compañía. En función de las combinaciones lingüísticas y de la temática, dichos sistemas pueden alcanzar niveles de rendimiento adecuados, aunque su coste puede ser elevado y requieren una importante inversión inicial de tiempo y recursos humanos. En el segundo caso, los sistemas gratuitos (como Google o DeepL) tienden a resultar menos útiles en contextos profesionales en cuanto no han sido adaptados a las necesidades de la empresa. Además, salvo excepciones (como la instalación fuera de línea en los casos de Apertium y de Lucy), a menudo los sistemas de TA gratuitos accesibles por navegador web no garantizan la confidencialidad de la información introducida. En cualquier caso, existen varios programas y toolkits con licencia libre y de código abierto, como Moses (TA estadística), MTradumàtica (TA estadística basada en Moses) o Apertium (TA basada en reglas), que permiten la creación o la adaptación de motores de TA a las necesidades específicas del usuario a partir de sus propios corpus paralelos (como memorias de traducción o colecciones de textos con licencias libres disponibles en internet) o en función de sus combinaciones lingüísticas.

Como recoge el Libro Blanco: título de grado en traducción e interpretación (Varios autores -ANECA- 2004), la competencia tecnológica es una de las competencias que deben desarrollar los grados en traducción e interpretación, y debe ser especificada en cuanto a contenidos para cada titulación. En España 
la formación de traductores se adaptó con relativa rapidez a la implantación de tecnologías en general y de programas de TAO en el mercado de trabajo, de manera que, en mayor o menor profundidad, los planes de estudios incluyen la formación correspondiente, tanto en el nivel de grado (Piqué \& Colominas 2013) como en el nivel de posgrado (Veiga Díaz 2013). Sin embargo, faltan estudios sobre la incorporación de la formación en TA y PE.

La implantación de las tecnologías de la traducción en la formación de traductores ha comportado la necesidad de que los centros se pregunten qué conocimientos deberían adquirir los estudiantes sobre las herramientas o con qué tipos de herramientas se debería trabajar en las aulas (Bowker 2015). La primera pregunta se plasma en el dilema teoría versus práctica (O’Brien \& Kenny 2011). En general las respuestas apuntan al hecho de que las herramientas evolucionan rápidamente, y por lo tanto es preferible que los estudiantes adquieran los principios generales de las diferentes clases de herramientas. Por otra parte, también se destaca la necesidad de que se ofrezca la oportunidad de practicar con diferentes herramientas de cada clase. La cuestión sobre la clase de herramientas se plantea en dos ámbitos principales. El primero es de carácter económico, ya que si las universidades quieren ofrecer formación en una variedad de programas determinados deben hacer frente al coste de las licencias (Flórez \& Alcina 2011); es por ello por lo que diferentes autores defienden el uso de software libre (Cánovas \& Samson 2011; Flórez \& Alcina 2011). El segundo ámbito se refiere a las categorías de herramientas que deberían ser objeto de la formación. Desde una perspectiva amplia, las herramientas que pueden ser relevantes para el traductor abarcan desde los programas de tratamiento de textos hasta los sistemas de traducción automática pasando por los diccionarios electrónicos, los sistemas de gestión de terminología, las herramientas de TAO, o las herramientas de gestión de proyectos, por mencionar solo algunas. Otras clases de herramientas se consideran más propias de la formación especializada, como los programas de subtitulación o de doblaje. A estas herramientas deben sumarse otras nuevas, como, por ejemplo, las plataformas de traducción colaborativa que combinan gestión terminológica, memorias de traducción, traducción automática, así como gestión del flujo de trabajo y del proyecto (O'Brien 2011: 19). Si se tiene en cuenta que 'empleabilidad' es una palabra clave en el diseño de planes de estudios en el Espacio Europeo de Educación Superior (EEES), parece obvio que los centros de formación de traductores deben preguntarse hasta qué punto la preparación que ofrecen en TA y PE responde a las exigencias que el mercado de trabajo plantea actualmente y planteará en el futuro a los egresados. 
Los objetivos de nuestro trabajo son dilucidar qué perfiles y competencias demanda el mercado de trabajo a los traductores en relación con la TA y la $\mathrm{PE}$, qué lugar ocupan la TA y la PE en la formación que ofrecen los grados y másteres de traducción de las universidades españolas y explorar un marco que permita determinar los perfiles y competencias correspondientes. Para ello en el apartado 2 presentamos una revisión de los estudios realizados sobre el uso de la TA y la PE entre los empleadores; su visión se complementa con el punto de vista de los profesionales acerca de la formación recibida y de las competencias más útiles. En el apartado 3 presentamos la metodología y los resultados de nuestro análisis de las páginas web de los grados y másteres en traducción de las facultades españolas; nos hemos centrado en perfiles, competencias y contenidos relacionados con la TA y la PE. En el apartado 4 exploramos un marco de perfiles y competencias basado en el modelo del proceso de traducción de Martín-Mor, Piqué \& Sánchez-Gijón (2016).

\section{Traducción automática y posedición en el mercado laboral}

El nuevo perfil profesional de los traductores puede situarse en el marco del paradigma que ha traído consigo el desarrollo de internet (Vargas Sierra \& Ramírez Polo 2012) con tres rasgos significativos: la facilidad de acceso a la información, nuevas formas de trabajo colaborativo y la necesidad de nuevas formas de especialización en el ámbito de los recursos tecnológicos. Varios estudios contribuyen a dibujar el perfil profesional de los traductores en la actualidad. Sin embargo, como veremos, aunque la conclusión de dichos estudios apunta hacia una presencia cada vez mayor de la TA y la PE en el sector, de esta conclusión no se desprende una definición clara de perfiles y competencias relacionadas con la TA y la PE.

\subsection{TA y PE en las empresas de servicios lingüísticos}

Entre los estudios de ámbito internacional, por orden cronológico podemos mencionar una ambiciosa encuesta encabezada por Rinsche \& Portera-Zanotti (2009) que, si bien se centraba en el tamaño de las industrias de la lengua, incluía preguntas acerca del uso de las tecnologías. De los 562 encuestados que respondieron las preguntas sobre tecnología, solo 36 (un 3,26\%) afirmaron utilizar TA. Son destacables también los resultados de una encuesta llevada a cabo por TAUS (Translation Automation User Society) entre empresas de servicios lingüísticos de Europa, América y Asia para conocer el uso de posedición de traducción automática: de las 75 empresas que respondieron a la encuesta, casi la mitad $(49,3 \%)$ ofrecían servicios de posedición de traducción automática 
(Joscelyne \& Brace 2010). En un estudio llevado a cabo por TradOnline entre profesionales (Varios autores - TradOnline-2011) se preguntaba si la tecnología comportaría cambios significativos en su manera de trabajar. De los 1.330 traductores que respondieron, el $48 \%$ pensaba que la tecnología cambiaría su manera de trabajar, aunque no de forma significativa; solo el $22 \%$ creía que los cambios serían importantes. Cabe destacar también el estudio llevado a cabo por Zaretskaya, Corpas Pastor \& Seghiri Domínguez (2015) con 736 traductores profesionales, en el que un 36\% de los encuestados manifestaron que utilizaban TA. El informe The Languages Services Market (DePalma, Hegde, Pielmeier \& Stewart 2014) mostraba que el 38,63\% de los encuestados ofrecía servicios de TA en un marco general de crecimiento del 6,23\% anual de la traducción. En la misma línea, los resultados del estudio MT Market Report 2014 de TAUS (Van der Meer \& Ruopp 2014) señalaban que el uso de la TA crecería en el futuro y que su desarrollo se integraría con otras tecnologías de la traducción como las herramientas de TAO. Este último estudio reviste un interés especial porque para su realización se entrevistó no solo a empresas de servicios lingüísticos sino también a desarrolladores de TA, usuarios, empresas comercializadoras de TA y consultores. Es el único trabajo de nuestros antecedentes que incluye un abanico tan amplio de perfiles de encuestados.

En España merece ser destacado por su amplitud el Estudio de situación del mercado español de servicios de traducción impulsado por la ACT, la Agrupación de Centros Especializados en Traducción (Varios autores - ACT_ 2005). En él y bajo el epígrafe 'tecnologías', se analizaba el uso de herramientas de TAO y de sistemas de TA, pero también de sistemas de maquetación y autoedición, así como de sistemas multimedia. En el año de la recogida de datos, 2004, solo el 10,91\% de las empresas usaba sistemas de TA. La baja implantación de TA como tendencia se mantendría aún diez años más tarde (Bel \& Rigau 2015), si bien esta baja implantación contrasta con las previsiones de crecimiento de las tecnologías del lenguaje en general, especialmente para la lengua española.

En 2015 el Grupo Tradumàtica de la UAB llevó a cabo una encuesta entre empresas de servicios lingüísticos españolas con el objetivo de analizar el grado de implantación de la TA y la PE en su actividad (Presas, Cid-Leal \& TorresHostench 2016). De las 55 empresas que respondieron la encuesta, un 52,7\% declaró no utilizar TA frente al 47,3\% que afirmó usarla, pero incluso en éstas su uso efectivo era muy bajo: casi la mitad de las empresas (45,5\%) utilizaban TA para un máximo del 10\% de sus proyectos. El $80 \%$ de las empresas confirmaban ofrecer servicios de PE, aunque en el $47 \%$ esta tarea correspondía a menos del $10 \%$ del volumen de trabajo. Por último, también se obtuvieron datos sobre la actitud de los traductores hacia la posedición; las respuestas 
mostraron que esta tarea despertaba cierto rechazo por su parte. Algunos de estos resultados sorprendieron al Grupo Tradumàtica. Por ello el estudio cuantitativo se complementó con un estudio cualitativo para el que se llevó a cabo una sesión de grupo focal con expertos. Por falta de espacio, aquí nos ceñiremos solo a dos de estos aspectos, relacionados con la desconfianza o el rechazo hacia el uso de la TA y la PE. Los participantes adujeron como posibles motivos ciertos prejuicios de los traductores que consideran que la TA está provocando cambios en su oficio, pero también, y seguramente de modo más significativo, la falta de formación de los traductores y las dificultades de la empresa para asumir los costes de implantación de un sistema propio de TA y la formación correspondiente de sus profesionales. Ambos argumentos se relacionarían con la calidad: para obtener una buena calidad es preciso que el traductor domine el sistema. Por lo que respecta a las reticencias hacia la $\mathrm{PE}$, los expertos opinaban que posiblemente irán disminuyendo a medida que el uso de la TA se difunda. Añadieron que con toda probabilidad las personas que ponen mayores obstáculos son las de más edad, frente a los jóvenes que mantienen una posición más abierta. No obstante, la ausencia de reticencias no era interpretada como algo necesariamente positivo: en algunos casos puede obedecer, sin más, a la ignorancia o falta de experiencia del traductor novel, que no conoce a ciencia cierta los problemas de calidad que puede conllevar la TA. Es de destacar que entre los expertos había consenso en que, desde un punto de vista económico, el uso de la TA en la empresa incrementa la productividad a largo plazo, aunque supone un coste inicial de implantación.

\subsection{La TA y la PE en la práctica profesional}

En conjunto observamos que los estudios reseñados en el apartado 2.1 muestran que la TA es considerada por desarrolladores e instituciones como la herramienta del futuro, pero que dista de ser percibida como una oportunidad por la mayoría de empresas y profesionales. En este apartado complementamos este panorama con la visión de profesionales acerca de las competencias que pueden considerarse más útiles o más demandadas. El programa OPTIMALE (Optimising Professional Translator Training in a Multilingual Europe), que se llevó a cabo entre 2010 y 2013, tenía el objetivo general de mejorar la visibilidad y la relevancia profesional de la formación de traductores en el nivel universitario en Europa (Varios autores —OPTIMALE- 2013). Entre otros objetivos parciales se proponía determinar tanto las competencias que la industria requería en aquel momento, como las competencias emergentes. Una de las actuaciones que llevó a cabo para ello fue una encuesta (abierta entre abril y octubre de 2012) que obtuvo 680 respuestas de empleadores. Los 
resultados de la encuesta debían servir de orientación tanto a los graduados que buscaban empleo como a los coordinadores de programas de formación de traductores interesados en incrementar la empleabilidad de sus egresados. En lo que respecta a las competencias relacionadas con la TA y la PE, los resultados muestran que el $76 \%$ de las empresas consideraban las habilidades de trabajar con memorias de traducción 'esenciales' o 'importantes'; el porcentaje se reducía a un $28 \%$ cuando se preguntaba por la posedición y caía al $18 \%$ para habilidades relacionadas con la preedición de textos para TA (Varios autores -OPTIMALE- 2012).

En 2014 la Conférence Internationale Permanente d'Instituts Universitaires de Traducteurs et Interprètes (CIUTI) lanzó una encuesta a egresados de programas de traducción. Respondieron el cuestionario 2.813 profesionales formados en 42 centros correspondientes a 19 países (Schmitt, Gerstmeyer $\&$ Müller 2016). Entre los datos relacionados con el uso de TA se puede destacar que el 10\% manifestaba usar algún sistema; entre éstos, el 70\% usaban Google Translate y menos del 20\% usaban un programa propio. Casi el 50\% opinaba que en la formación de traductores debería prestarse más atención a las herramientas tecnológicas.

En 2015 el European Master's in Translation (EMT) llevó a cabo un estudio en el que participaron más de 1.700 egresados de universidades europeas, los resultados del cual fueron recogidos por Krause (2017: 150). Su objetivo era identificar cuáles de las competencias adquiridas en la universidad resultaban más útiles en el ámbito profesional. Un 38\% valoraba positivamente el dominio de herramientas de TAO y un $13 \%$ valoraba positivamente competencias relacionadas con posedición. Sorprende que no se incluyera ninguna pregunta relacionada específicamente con conocimientos de TA. Como es sabido, el grupo de expertos del EMT proporciona periódicamente una actualización de su marco de competencias. En la última (Varios autores -EMT- 2017) sitúa las competencias en cinco áreas: lengua y cultura, traducción, tecnología, personal e interpersonal y provisión de servicio. En el área de tecnología encontramos dos competencias relacionadas con TA: dominar los conceptos básicos de la TA y su impacto en el proceso de traducción, por un lado, y determinar la relevancia de los sistemas de traducción automática en el flujo de trabajo e implementar los sistemas de TA apropiados cuando sea necesario por el otro.

De todo ello se puede concluir con Krause (2017: 155) que un programa de traducción orientado al futuro debería ofrecer asignaturas específicas de traducción automática, preedición y posedición, control automático de calidad y gestión de proyectos. 


\section{La TA y la PE en la formación de traductores}

En el contexto descrito en el apartado anterior, los programas de formación de traductores tienen ante sí el reto de determinar qué competencias relacionadas con la TA y la PE deben adquirir los estudiantes, con qué herramientas deberían trabajar, en qué estadios de la formación deberían introducirse, y en qué asignaturas. Dicho de otra manera, deben concretar para la TA y la PE las competencias tecnológicas específicas que definía de forma genérica el Libro Blanco (Varios autores —ANECA- 2004: 85): 'Uso de herramientas informáticas' y 'Dominio de técnicas de traducción asistida/localización'.

Como ponían de manifiesto los estudios ya mencionados de Veiga Díaz (2013) y Piqué \& Colominas (2013), en ese momento en los programas de grado y posgrado de las universidades españolas la formación en herramientas de traducción se centraba en el uso de recursos de TAO y de localización. Parecía necesario analizar hasta qué punto, cinco años más tarde, los grados y másteres de traducción que se ofrecen en España se han actualizado y han incorporado la TA y la PE en sus perfiles, competencias y contenidos. Con nuestro análisis hemos tratado de responder a las siguientes preguntas: ¿identifican un perfil específico relacionado con la TA y la PE?, ¿se proponen desarrollar competencias relacionadas con la TA y la PE? y ¿ofrecen contenidos y metodología relacionados con la TA y la PE?

\subsection{Metodología}

Nuestro análisis de los sitios web se justifica por dos razones básicas. En primer lugar, porque las universidades españolas están obligadas a publicar sus planes de estudios y guías docentes, por lo que los sitios web ofrecen un material significativamente amplio y diverso al investigador. En segundo lugar, porque sirven para mostrar al futuro estudiante qué orientación y qué salidas profesionales tienen los estudios que desea emprender. La revisión se efectuó durante el primer semestre del curso 2017-2018.

En total se han analizado las páginas web de 23 grados de 26 universidades (tres de las cuales comparten un grado) y 22 másteres de 19 universidades (tres de las cuales comparten un mismo máster) dedicados a la formación de traductores. Se han descartado los másteres de traducción literaria e interpretación. La lista de partida de titulaciones corresponde a las recogidas en la Conferencia de Centros y Departamentos de Traducción e Interpretación (CCDUTI) en su reunión de 2017. Además, se ha hecho una búsqueda complementaria para garantizar la mayor exhaustividad posible. 
Para cada titulación (grados y másteres) se ha recogido la formulación general de competencias y perfiles profesionales tal y como aparecen en las páginas web.

Además, en el caso de los grados, se han revisado las guías docentes de asignaturas básicas, obligatorias y optativas, centradas en el uso de herramientas de traducción. En general, las guías tienen los mismos apartados: presentación, competencias, resultados de aprendizaje, contenidos, actividades formativas o metodología, sistema de evaluación y bibliografía. No obstante, los datos de cada apartado son más o menos detallados según la universidad y la titulación. De las guías de las asignaturas se han recogido los elementos descriptivos (denominación, tipo, curso y número de créditos), enunciado explícito de competencias, contenidos y metodología de TA y/o PE.

En los másteres también se han revisado las guías docentes. Aquí no podemos hablar de asignaturas porque los másteres responden a diversas estructuras. Así, por ejemplo, en algunas universidades la formación se estructura en módulos que a su vez se dividen en contenidos, a los cuales se denomina o no 'asignaturas'. En otras no hay módulos, solo hay asignaturas obligatorias y optativas. En otras los contenidos/asignaturas se dividen en 'especialización', 'especialidad', 'itinerario', 'bloque de asignaturas' o 'mención'. Esta diversidad terminológica ha dificultado la sistematización y el análisis de los datos; para una mejor comprensión del lector, en este trabajo se utiliza la denominación 'asignatura'. En estas asignaturas se imparten contenidos relacionados con herramientas de traducción, traducción especializada (comercial, administrativa, jurídica, económica, financiera, científica, técnica, médica, institucional), traducción audiovisual y localización. En aquellos casos en los que una misma asignatura o contenido se imparte en diferentes pares de lenguas se ha analizado un solo par, normalmente el inglés-español. Al igual que en los grados, de estas guías se han recogido los elementos descriptivos (denominación, tipo, curso y número de créditos) y el enunciado explícito de competencias, contenidos y metodología de TA y/o PE.

En total se han recopilado datos de 42 asignaturas de grado. En cuanto a los másteres, se han identificado 167 asignaturas, de las cuales 43 no publican las guías docentes, por lo que el cómputo final es de 124 guías analizadas. En resumen, se han revisado, para grados y másteres:

- Los enunciados de las competencias generales y específicas de la titulación.

- Los enunciados de los perfiles profesionales generales de la titulación.

- Los elementos descriptivos de la asignatura: denominación, tipo, curso y número de créditos. 
- Enunciado explícito de competencias de TA y/o PE de la asignatura.

- Enunciado explícito de contenidos de TA y/o PE de la asignatura.

- Enunciado explícito de metodología de TA y/o PE de la asignatura.

\subsection{Resultados}

En lo que respecta a los grados, los enunciados de las competencias generales y/o específicas mencionan 'recursos tecnológicos', 'herramientas informáticas', 'traducción asistida', 'localización', 'TIC aplicadas a la traducción'. Solo una universidad menciona la TA como ejemplo de TIC y ninguna menciona la PE. Ningún perfil profesional alude a salidas profesionales relacionadas explícitamente con TA y/o PE.

En cuanto a las 42 asignaturas analizadas, solo tres incluyen explícitamente TA o PE en sus denominaciones: 'Traducción automática y asistida', 'Traducción automática y posedición', 'Posedición'. En el resto aparecen términos como 'herramientas', 'tecnologías', 'recursos tecnológicos' o 'informática', de forma genérica. Ocho son optativas y el resto son básicas u obligatorias. Se imparten preferentemente en los cursos $1 .^{\circ}, 2 .^{\circ}$ y $3 .^{\circ}$. La mayoría son de 6 créditos.

El análisis de las guías de las asignaturas pone de manifiesto que solo 10 mencionan explícitamente la TA y 4 la PE en los enunciados de sus competencias. Con respecto a los contenidos, 15 asignaturas incluyen la TA y 5 la PE. Por último, en la metodología 3 asignaturas contemplan la TA y 3 la PE. Cabe remarcar no obstante que una asignatura puede incluir TA y/o PE en sus contenidos, pero no vincular su metodología con ellos, incluso no aparecer ninguna competencia específica. La ausencia de metodología vinculada a los contenidos de TA y PE impide saber si se explican como tareas independientes o una vinculada a la otra. La figura 1 resume lo explicado en los párrafos anteriores:

\begin{tabular}{|c|c|c|}
\hline $\begin{array}{c}\text { Mención explícita de } \\
\text { TA y/o PE en el: }\end{array}$ & TA & PE \\
\hline $\begin{array}{c}\text { Enunciado de las } \\
\text { competencias }\end{array}$ & 10 asignaturas (23,8\%) & 4 asignaturas $(9,5 \%)$ \\
\hline $\begin{array}{c}\text { Enunciado de los } \\
\text { contenidos }\end{array}$ & 15 asignaturas (35,7\%) & 5 asignaturas $(11,9 \%)$ \\
\hline $\begin{array}{c}\text { Enunciado de la } \\
\text { metodología }\end{array}$ & 3 asignaturas $(7,1 \%)$ & 3 asignaturas $(7,1 \%)$ \\
\hline
\end{tabular}

Figura 1. La TA y la PE en las competencias, contenidos y metodología de las asignaturas de grado. 
Con respecto a los másteres, solo dos titulaciones incluyen explícitamente en sus competencias generales y/o específicas la TA y solo una la PE.

La mención de la TA y la PE como perfil profesional solo se encuentra en una titulación: 'Traductor especializado en tecnologías de la traducción (uso de TA, MT, gestores terminológicos, edición de textos, etc.)'. En el resto, se hace referencia general a las tecnologías y herramientas necesarias para llevar a cabo las tareas de traducción, sin más especificación.

En las guías docentes de las 124 asignaturas se observa que 4 de ellas citan explícitamente la TA como una competencia específica y 9 la PE. En relación con los contenidos, 6 incluyen TA y 3 PE. Ninguna asignatura incluye la TA y/o la PE en su metodología (ver figura 2). De nuevo, la ausencia de descripción de la metodología impide saber si la TA y la PE se enseñan como tareas interconectadas o no.

\begin{tabular}{|c|c|c|}
\hline $\begin{array}{c}\text { Mención explícita de } \\
\text { TA y/o PE en el: }\end{array}$ & TA & PE \\
\hline $\begin{array}{c}\text { Enunciado de las } \\
\text { competencias }\end{array}$ & 4 asignaturas (3,2\%) & 9 asignaturas (7,2\%) \\
\hline $\begin{array}{c}\text { Enunciado de los } \\
\text { contenidos }\end{array}$ & 6 asignaturas (4,8\%) & 3 asignaturas $(2,4 \%)$ \\
\hline $\begin{array}{c}\text { Enunciado en la } \\
\text { metodología }\end{array}$ & 0 asignaturas & 0 asignaturas \\
\hline
\end{tabular}

Figura 2. La TA y la PE en las competencias, contenidos y metodología de las asignaturas/ contenidos de máster.

En síntesis, los datos recogidos permiten responder a las preguntas que se planteaban al inicio de este apartado:

- En general puede afirmarse que ni los grados ni los másteres identifican un perfil profesional específico relacionado con la TA y la PE.

- Las competencias relacionadas con la TA y la PE tienen una presencia relativamente baja en los grados y puramente testimonial en los másteres.

- Los grados tienen más contenidos de TA y PE en los enunciados y en la metodología que los másteres.

Los resultados de nuestro estudio indicarían una presencia muy baja de la TA y la PE en la formación de traductores en las universidades españolas. Sin embargo, hay que tener en cuenta que es posible que el profesorado de 
tecnologías sí que las incluya en los programas de sus asignaturas bajo epígrafes genéricos como 'herramientas informáticas' o 'recursos tecnológicos'.

\section{Perfil y marco de competencias de TA y PE}

No cabe duda de que la implantación de tecnologías específicas en el sector de la traducción ha impulsado y seguirá impulsando una redefinición de la profesión del traductor que, tal vez con excesivo optimismo, ha sido caracterizada como 'multilingual multimedia communication engineering' (Gouadec 2007: 123). Sí parece cierto, como apunta García (2010: 3), que en el sector de los servicios de traducción se observa un desplazamiento desde el estadio de la traducción humana asistida por ordenador hacia el estadio de la traducción automática con asistencia humana según el modelo propuesto por Hutchins \& Somers. La asistencia humana consiste en la participación interactiva durante el proceso o bien fuera de este, en las fases de preedición y posedición (Hutchins \& Sommers 1992: 150). Ante esta situación el traductor puede optar por especializarse en ámbitos donde la TA todavía no puede superar al traductor humano, o bien adquirir nuevas habilidades como pre y poseditor, asesor lingüístico o experto en aseguramiento de la calidad (García 2010: 6). Sin embargo, como se desprende de los estudios resumidos en el apartado 2 de este artículo, para que se produzca esta evolución es necesario que los profesionales superen sus reticencias hacia las tareas relacionadas con la TA y la PE. Puesto que estas reservas se deben en buena medida a la falta de formación de los traductores es necesario que los programas incorporen los perfiles y competencias relevantes. De hecho, como ponen de manifiesto Cadwell, O’Brien \& Teixeira (2017), si los profesionales se implican en el control de las herramientas, mejora su percepción acerca de ellas. Esta afirmación está en la línea del programa Science with and for Society, impulsado por la Comisión Europea en el marco de la estrategia de investigación Horizon 2020 (European Comission 2018); en dicho programa se propugna que investigadores, ciudadanos, responsables políticos, empresas, organizaciones del tercer sector y otros agentes sociales colaboren en todo el proceso de investigación e innovación, con el objetivo de reducir la brecha entre la comunidad científica y la sociedad.

Diferentes trabajos se han ocupado de definir las competencias tecnológicas de los traductores en general, como los de Austermühl (2013), Doherty \& Moorkens (2013), Enríquez Raído (2013), Samson (2013) o Torres del Rey (2005) por citar solo algunos. También se han presentado propuestas para la formación de poseditores (Rico \& Torrejón 2012; Sánchez-Gijón 2016; Varios autores - ISO - 2017) y para la formación en TA (Kenny \& Doherty 2014; 
Doherty \& Kenny 2014; Rico 2017). En este apartado revisamos estas propuestas y presentamos nuestro propio marco que integra perfiles y competencias de los dos ámbitos de actividad.

\subsection{Perfiles y competencias de TA}

Las propuestas de competencias de TA parten de la reflexión sobre la relación entre el traductor y la tecnología y tratan de evitar los enfoques que reducen el papel de este al de mero revisor.

Así, por ejemplo, Kenny \& Doherty (2014: 290) abogan por una aproximación holística y proactiva de la enseñanza y aprendizaje de la TA, que se concreta en cinco competencias que combinan TA y TAO (Doherty \& Kenny 2014: 299): demostrar comprensión de los principios de las tecnologías de TAO; usar al menos una herramienta de TAO comercial; demostrar comprensión de los principios de la TA actual, incluida la TA basada en reglas y la TA estadística; gestionar flujos de trabajo completos relacionados con TAO y TA; evaluar críticamente tecnologías de la traducción actuales y los textos producidos con estas.

Rico (2017: 76) constata que el objetivo de la industria de obtener máxima productividad a precios mínimos y con calidad máxima puede llevar a un empobrecimiento de la labor del traductor humano, que se limitaría a revisar segmentos aislados de una traducción producida por un programa informático. En contraposición, la autora propone revisar el papel de la tecnología en el proceso de traducción analizando la interacción que se da entre esta y el usuario, y defiende que la formación en TA debe dotar al traductor de las competencias y capacidades necesarias para controlar el proceso (Rico 2017: 89). A partir de un modelo cíclico del proceso de traducción establece dos grupos de competencias relacionadas con la TA como proceso y con la TA como producto. En el primero se agrupan competencias que reflejan la relación que se establece entre el usuario y la máquina. En el segundo, agrupa competencias que tradicionalmente se han incluido en el concepto de competencia tecnológica, como, por ejemplo, el dominio de diferentes tipos de sistemas de TA como usuario avanzado o el conocimiento de técnicas de PE (Rico 2017: 86).

\subsection{Perfiles y competencias de PE}

Sánchez-Gijón (2016) distingue dos perfiles relacionados con la PE. El primero es más limitado, pero es el que está más consolidado en los servicios lingüísticos; consiste en editar y validar las propuestas de traducción obtenidas con un programa de TA. El segundo perfil comparte con el primero la edición de 
segmentos de TA, pero amplía las funciones del traductor, que consistirán en editar segmentos de TA, preparar materiales de PE y, en general, gestionar sistemas de TA. La diferencia entre ambos perfiles viene marcada por los diferentes grados de dominio de la tecnología. La edición de segmentos requiere únicamente el uso de herramientas de edición siguiendo parámetros preestablecidos. La preparación de materiales de PE implica interactuar con un sistema de TA e incluso elaborar recursos lexicográficos o terminográficos. Por último, la gestión de sistemas de TA implica tareas como la creación u optimización de motores de TA estadística o bien la modificación y mejora de sistemas de TA basados en reglas (Sánchez-Gijón 2016: 156-157).

Como autor representativo de la primera concepción podemos señalar a Pym (2013: 493), que observa que la función del traductor se ha desplazado de la generación de soluciones para los problemas de traducción a la selección de soluciones que presenta el programa informático. Llevando esta concepción al extremo, la competencia del poseditor/traductor se reduciría básicamente a la competencia lingüística y a la capacidad de cumplir un encargo con 'confianza justificada' (Pym 2003: 489).

En cambio, Rico \& Torrejón (2012: 169-170) definen las competencias del poseditor desde el punto de vista más amplio. Los autores parten del supuesto de que la posedición es una profesión en ella misma. Las competencias se agrupan en tres categorías: competencias nucleares, habilidades lingüísticas y competencias instrumentales. Las competencias nucleares son de carácter actitudinal y estratégico. La competencia actitudinal permite al poseditor gestionar la subjetividad y las incertidumbres a la hora de definir y aplicar especificaciones de PE o gestionar las expectativas del cliente en cuanto a la calidad del texto. La competencia estratégica ayuda al poseditor a adoptar decisiones informadas en el momento de elegir entre soluciones alternativas. Por otro lado, las competencias instrumentales incluyen conocimientos sobre TA, gestión de terminología, actualización de diccionarios de memorias de traducción y de sistemas de TA y destrezas básicas de programación. Los autores, además, integran las tareas de PE en el marco de un proyecto de traducción en el que son relevantes la descripción del cliente, la descripción del texto o el motor de traducción automática entre otros factores. En síntesis, describen un perfil profesional que puede gestionar sistemas de TA, interactuar con herramientas y recursos de traducción según las necesidades del proyecto y además gestionar cualquier tarea de un proyecto de traducción.

En la misma línea, Sánchez-Gijón (2016) presenta al traductor como un profesional que no solo se limita a elaborar un texto en la lengua final, sino que también puede intervenir en todas las fases del proceso y que, además, 
es capaz de elegir diferentes estrategias según el encargo de traducción. Así pues, el traductor/poseditor podría participar, gestionar y/o ejecutar tanto un proyecto de traducción como uno de posedición ya sea individual o grupal.

También en la norma ISO18587:2017 (Varios autores _ISO_ 2017: 9) se enfatiza la formación tecnológica del poseditor. Sus autores, además de competencias de traducción y documentación, lingüísticas, culturales y temáticas, requieren un dominio avanzado de herramientas de TA y de TAO, de bases de datos terminológicas y de procesadores de textos, así como práctica en ambos tipos de posedición y en el uso de herramientas de control de calidad. Este modelo parte de la especificación de las tareas que debe llevar a cabo el poseditor: leer el resultado de la TA y evaluar si es necesario reformularlo; comprender el contenido en lengua original para utilizarlo como referencia y llevar a cabo las correcciones necesarias; producir un contenido en lengua meta ya sea utilizando como base elementos del resultado de la TA o bien proporcionando una nueva traducción.

\subsection{Perfil y competencias de TA y PE}

En el modelo pedagógico del Espacio Europeo de Educación Superior (EEES) una competencia se define como un 'saber actuar complejo' (Lasnier 2000: 32), no en general, sino en 'una clase de situaciones comparables' (Perrenoud 2005: 3). Así pues, formular perfiles y competencias implica no solo definir los contenidos teóricos (saber qué) o las habilidades prácticas (saber cómo) que deberán adquirir los estudiantes, sino, sobre todo, a qué situaciones podrán aplicarse (saber por qué). Los modelos analizados en los apartados 4.1 y 4.2 definen las competencias a partir de alguno de estos factores, pero a nuestro juicio no integran los tres factores en cada una de las competencias.

Nuestra propuesta intenta resolver el dilema teoría versus práctica y tiene en cuenta las fases y tareas del proceso, así como sus objetivos - las situaciones-, las tareas que deben llevarse a cabo en cada fase - habilidades prácticas- y los conocimientos sobre las herramientas necesarias en cada fase - conocimientos teóricos-. El dominio de estas competencias debería situarse en el nivel de experto, que se caracteriza por una ejecución buena y eficaz, sin necesidad de control externo (Pozo 1996). En esta propuesta general dejamos de lado factores tan importantes de cada situación concreta como el par de lenguas, el ámbito de conocimiento del texto o su grado de especialización (Presas 2008). Por otra parte, las competencias se orientan a potenciar el trabajo con sistemas de TA propios, como propugnan Gaspari, Almaghout \& Doherty (2015). 
La propuesta parte de un modelo de digitalización del proceso de traducción (Martín-Mor, Piqué \& Sánchez-Gijón, 2016) que concibe este proceso como una secuencia de fases a las que corresponden diversas tareas, si bien dicha secuencia puede variar en función de cada encargo de traducción. El modelo refleja las tareas comunes en el trabajo del traductor con herramientas de TAO y de TA, así como las tareas distintivas de cada tipo de herramienta (ver figura 3). Con ello, desde el punto de vista de la formación de traductores se integra la posibilidad de transferencia de conocimientos entre ambas tecnologías, pero también se permite la definición de perfiles específicos. Por último, el modelo sitúa las tareas que podemos considerar como propiamente de traducción en el contexto más general del flujo de trabajo. Así, la primera fase, 'obtención de la traducción', y la última, 'entrega del trabajo', se caracterizan por estar dedicadas a la gestión de los archivos y a la comunicación con el cliente. En la fase de 'análisis' se agrupan las tareas que tienen como objetivo recopilar la información necesaria sobre el texto final con el fin de determinar qué tareas deberán llevarse a cabo y el tiempo que requerirán. En la fase de 'preparación' se recopilan los materiales que se utilizarán en la fase de 'traducción inicial'. En la fase de 'postproducción' se llevan a cabo las tareas de corrección y optimización.

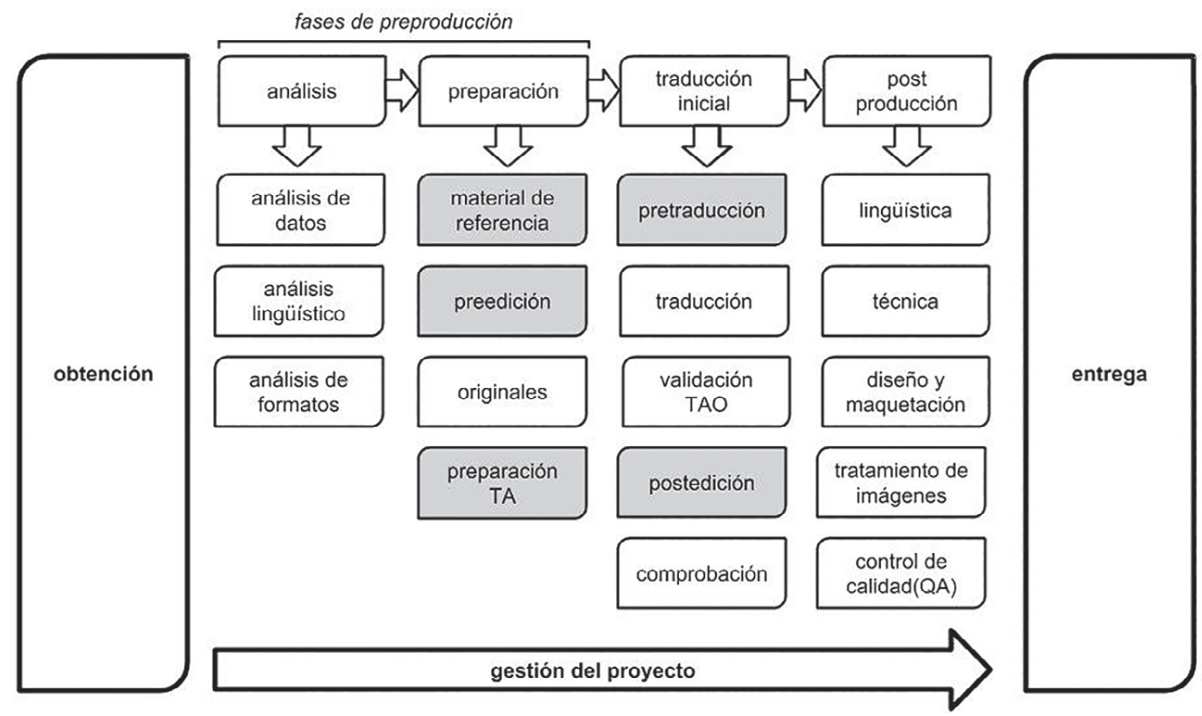

Figura 3. Modelo de digitalización del proceso de traducción (Adaptado de: Martín-Mor, Piqué, Sánchez-Gijón 2016: 34). Sombreadas en gris aparecen las tareas específicas de TA y PE. 
En este modelo, las tareas específicas de TA corresponden a las fases de 'preparación' y 'traducción inicial'. El modelo describe de manera pormenorizada las tareas, funciones y herramientas que forman parte de cada fase:

- La fase de 'preparación' incluye las tareas de preparación de materiales de referencia (gestión de terminología y corpus), preedición, preparación de originales y preparación de TA. La preparación de material de referencia consiste en la creación y configuración de materiales tales como bases de datos terminológicas, memorias de traducción y directrices lingüísticas, que se utilizarán en la fase de traducción. La preedición de textos consiste en modificar el texto original para la traducción automática. Incluye la reducción de ambigüedades propias del lenguaje natural o la simplificación de la sintaxis del texto, normalmente mediante la aplicación de guías específicas. En esta fase se pueden utilizar procesadores de textos y gestores terminológicos con el fin de automatizar el trabajo. La preparación de motores de TA consiste en la creación o configuración de los motores que se utilizarán durante la traducción. Por ejemplo, en el caso de traducción automática estadística (TAE), puede ser necesario entrenar el motor con nuevos textos, en el caso de la traducción automática basada en reglas (TABR) puede ser necesario añadir nuevas reglas para resolver problemas concretos.

- En la fase de 'traducción inicial' se sitúan las tareas de pretraducción, traducción, validación de la TAO, posedición y comprobación. Las específicas de la TA son pretraducción y posedición. En la fase de pretraducción se obtiene un texto en bruto, conseguido a partir de un motor de TA, que puede considerarse como una sucesión de oraciones que serán el punto de partida de la posedición. La tarea más importante que se lleva a cabo en esta fase es estimar la calidad del texto en bruto a partir de una muestra. Para ello se utilizan diversos tipos de procedimientos y herramientas (métricas). La posedición es la última de las tareas relacionada con la TA en la fase de traducción inicial y consiste esencialmente en corregir la traducción en bruto con el fin de obtener un texto que presente un nivel de calidad que ha sido determinado previamente.

La formulación de las competencias y contenidos concretos de un plan de estudios es atribución de las facultades, por lo que aquí nos limitaremos a presentar un marco general sin especificar qué competencias son más propias de un grado o de un máster en traducción. En nuestra propuesta asociamos 
las competencias con la clase de situaciones en que deberían poder aplicarse -las tareas específicas - y con las categorías de herramientas que se utilizan para cada una de las tareas. Además, definimos las competencias de acuerdo con los tres tipos de conocimientos: los conocimientos condicionales (saber por qué), que se enuncian mediante el verbo 'comprender'; los conocimientos operacionales (saber cómo), que se enuncian mediante el verbo 'utilizar', y los conocimientos teóricos (saber qué), que se enuncian mediante los verbos 'conocer' y 'comprender'.

- Competencia de preparación de material de referencia. Incluye comprender el objetivo para el que servirán los materiales, esencialmente, corpus y bases de datos terminológicas; conocer herramientas de gestión de corpus y de gestión de terminología y comprender su función, tanto en la fase de preparación de materiales como en el flujo de trabajo; utilizar las herramientas para alinear corpus, extraer terminología, introducir nuevos términos o revisar glosarios.

- Competencia de preedición. Incluye comprender el objetivo y la necesidad de las tareas de preedición en función del sistema de TA que haya de ser utilizado; conocer y comprender las herramientas adecuadas para llevar a cabo tareas de preedición; utilizar las herramientas para reducir ambigüedades del lenguaje natural o simplificar la sintaxis.

- Competencia de preparación de motores de TA. Incluye comprender el objetivo y la oportunidad de utilizar motores de TA; conocer y comprender los distintos tipos de motores; entrenar motores para sistemas de TAE y de TA neural, así como modificar reglas para sistemas de TABR.

- Competencia de pretraducción. Incluye comprender el objetivo y la necesidad de estimar la calidad del producto de la TA; conocer y comprender los criterios de calidad, así como las herramientas que se utilizan para ello; estimar la calidad del texto en bruto mediante la herramienta adecuada.

- Competencia de posedición. Incluye comprender el objetivo y la necesidad de revisar el texto en bruto; conocer y comprender directrices y guías de PE, los niveles de calidad de la PE, así como recursos tecnológicos para llevar a cabo la tarea; modificar el texto en bruto mediante la herramienta adecuada. 


\section{Conclusiones}

El perfil del traductor que se busca en el ámbito laboral debe incorporar cada vez más competencias relacionadas con las tecnologías. El empleo de las tecnologías se redefine de forma continua de acuerdo con su propia evolución y con las necesidades del mercado.

La TA se considera como un sector estratégico que genera un volumen de negocio muy importante. Su uso trasciende el ámbito de las empresas de servicios lingüísticos y se utiliza ampliamente en nuevos sectores como la prensa escrita, la administración pública o los entornos editoriales.

La formación de traductores debe tener en cuenta los perfiles profesionales que favorecen la empleabilidad de sus egresados. De ahí la necesidad de conocer las demandas actuales del mercado de trabajo y prever su evolución.

Hemos constatado que la formación universitaria de traductores en España incluye asignaturas de herramientas tecnológicas para la traducción y que la mayoría de las mismas corresponden a formación básica u obligatoria. En los grados los contenidos se centran mayoritariamente en herramientas de TAO, mientras que la TA y la PE ocupan poco espacio en las programaciones docentes. Por otro lado, la TA y la PE están ausentes de las competencias generales de los grados y no se especifican perfiles o salidas profesionales vinculadas con ellas. La TA y la PE tienen todavía menor presencia en los planes de estudio de los másteres. Esta situación contrasta con las tendencias y demandas del mercado profesional. Para que los egresados puedan mejorar su grado de empleabilidad sería necesario que la TA y la PE tuvieran una mayor presencia en los planes de estudio, y que se incluyeran competencias y conocimientos relacionados con las fases y procesos del flujo de trabajo con TA y las tareas que puede llevar a cabo el traductor en dicho flujo.

Con este trabajo hemos obtenido una panorámica general sobre la presencia de la TA y la PE en la formación universitaria de traductores. Un estudio posterior entre el profesorado nos permitirá profundizar en aspectos concretos; por ejemplo, si epígrafes genéricos como 'herramientas informáticas' o 'recursos tecnológicos' incluyen la TA y la PE, en qué medida ambas se trabajan de forma integrada en las asignaturas específicas o en qué medida estas asignaturas específicas se coordinan con asignaturas de traducción. Asimismo, queda abierto para un posterior y necesario debate la concreción de aspectos de las competencias propuestas; por ejemplo, con qué herramientas se debería trabajar en el aula, la progresión en que deberían introducirse en la formación o en qué asignaturas. 


\section{Referencias bibliográficas}

AUSTERMÜHL, Frank. (2013) "Future (and not-so-future) trends in the Teaching of Translation Technology." Revista Tradumàtica: tecnologies de la traducció 11, pp. 326-337. Versión electrónica: <https://doi.org/10.5565/rev/tradumatica.46>

BEL, Núria \& German RigAu (eds.) (2015) Informe sobre el estado de las tecnologías del lenguaje en España dentro de la Agenda Digital para España. Madrid: Secretaría de Estado de Telecomunicaciones y para la Sociedad de la Información. Versión digital: <http://www.agendadigital.gob.es/tecnologias-lenguaje/ Bibliotecaimpulsotecnologiaslenguaje/Material\%20complementario/InformeTecnologias-Lenguaje-Espana.pdf>

BOWKER, Lynne. (2015) "Computer-Aided Translation: Translator training." En: The Routledge Encyclopedia of Translation Technology. Nueva York: Routledge, pp. 88-104.

CAdWEll, Patrick; Sharon O'Brien \& Carlos S.C. TeIXeIRA. (2017) "Resistance and accommodation: factors for the (non-) adoption of machine translation among professional translators." Perspectives 26:3, pp. 1-21. Versión electrónica: <https://doi.org/10.1080/0907676X.2017.1337210>

CÁnOvas, Marcos \& Richard SAMSON. (2011) "Open source software in translator training." Revista Tradumàtica: tecnologies de la traducció 9, pp. 46-56. Versión electrónica: <http://revistes.uab.cat/tradumatica/article/view/ n9-canovas-samson/pdf>

DePalma, Donald A.; Vijayalaxmi Hedge; Hélène Pielmeier \& Robert G. STEWART. (2014) The Language Services Market 2014. Cambridge, MA: Common Sense Advisory. Versión electrónica: <http://www.commonsenseadvisory. com/Portals/_default/Knowledgebase/ArticleImages/140625_R_Language_ Services_Market_2014_Preview.pdf>

DOHERTY, Stephen \& Dorothy KenNy. (2014) "The design and evaluation of a Statistical Machine Translation syllabus for translation students". The Interpreter and Translator Trainer 8:2, pp. 295-315. Versión electrónica: <https:doi.org/10 .1080/1750399X.2014.937571>

DOHERTY, Stephen \& Joss MOORKENS. (2013) "Investigating the experience of translation technology labs: pedagogical implications." The Journal of Specialised Translation 19, pp. 122-136. Versión electrónica: <http://doras.dcu.ie/19475/1/ art_doherty.pdf>

ENRÍQUEZ RAÍDO, Vanessa. (2013) “Teaching Translation Technologies 'Everyware': Towards a Self-Discovery and Lifelong Learning Approach." Revista Tradumàtica: tecnologies de la traducció 11, pp. 275-285. Versión electrónica: <https://doi. org/10.5565/rev/tradumatica.52> 
European Commission. (2018) Horizon 2020. Brussels: European Commission. Versión electrónica: <https://ec.europa.eu/programmes/horizon2020/en/ h2020-section/responsible-research-innovation>

FlóREZ, Silvia \& Amparo AlCiNA. (2011) "Free/open-source software for the translation classroom: A catalogue of available tools." The Interpreter and Translator Trainer 5:2, pp. 325-357. Versión electrónica: <https://doi.org/10.1080/1355 $6509.2011 .10798824 \geq$

GARCÍA, Ignacio. (2010) "The proper place of professionals (and non-professionals and machines) in web translation." Revista Tradumàtica: tecnologies de la traducció 8, pp. 1-7. Versión electrónica: <http://www.fti.uab.cat/tradumatica/ revista/num8/articles/02/02art.htm>

GASPARI, Federico; Hala Almaghout \& Stephen DOHERTY. (2015) "A survey of machine translation competences: Insights for translation technology educators and practitioners." Perspectives: Studies in Translatology 23:3, pp. 333-358. Versión electrónica: <http://dx.doi.org/10.1080/0907676X.2014.979842>

GOUADEC, Daniel. (2007) Translation as a profession. Ámsterdam [etc.]: John Benjamins.

HutCHINS, W. John \& Harold L. SOMERS. (1992) An introduction to machine translation. Londres: Academic Press.

JosCelyne, Andrew \& Colin Brace. (2010) Postediting in Practice: A TAUS Report. Versión electrónica: <https://www.taus.net/think-tank/reports/postedit-reports/ postediting-in-practice>

KENNY, Dorothy \& Stephen DOHERTY. (2014) "Statistical machine translation in the translation curriculum: overcoming obstacles and empowering translators." The Interpreter and Translator Trainer 8:2, pp. 276-294. Versión electrónica: <https:doi.org/10.1080/1750399X.2014.936112>

KRAUSE, Alexandra. (2017) "Program designing in translation and interpreting and employability of future degree holders.” En: Valero Garcés, Carmen \& Carmen Pena Díaz (eds.). AIEI 8: Superando límites en traducción e interpretación = Além dos limites em traduçao e interpretaçao = Beyond limits in Translation and Interpreting. Ginebra: Tradulex, pp. 147-158. Versión electrónica: <http://www. tradulex.com/varia/AIETI8.pdf>

LASNIER, Françoise. (2000) Réussir la formation par compétences. Montreal: Guérin. MARTín-Mor, Adrià; Ramon PiQUÉ \& Pilar SÁnCHEZ-GIJón. (2016) Tradumàtica: tecnologies de la traducció. Vic: Eumo.

O`BRIEN, Sharon. (2011) “Collaborative translation.” En: Gambier, Yves \& Luc van Doorslaer. Handbook of Translation Studies. Ámsterdam, Filadelfia: John Benjamins, vol. 2, pp. 17-21

O`BRIEN, Sharon \& Dorothy Kenny. (2011) "In Dublin Fair City: Teaching Technology at Dublin City University." Language International 13:5, pp. 20-23. 
Versión electrónica: <http://www.mt-archive.info/jnl/LangInt-2001-OBrien. pdf>

Perrenoud, Philippe. (2005) "Développer des compétences, mission centrale ou marginale de l'université?" En: XXIIème Congrès de l'Association International de Pédagogie Universitaire: L'enseignement supérieur du XXIe siècle: de nouveaux défi s à relever: du 12 au 14 septembre 2005 Université de Genève: Uni Mail, Université de Genève (Suisse). Ginebra: la Université.

PIQUÉ, Ramon \& Carme Colominas. (2013) "Les tecnologies de la traducció en la formació de grau de traductors i intèrprets." Revista Tradumàtica: tecnologies de la traducció 11, pp. 297-312. Versión electrónica: <https://doi.org/10.5565/ rev/tradumatica.43>

Pozo Municio, Juan Ignacio. (1996) Aprendices y maestros: la nueva cultura del aprendizaje. Madrid: Alianza.

PRESAS, Marisa. (2008) "Formar traductores expertos en el EEES: un marco para la formulación de competencias de los estudios de Grado." Sendebar 19, pp. 213-244. Versión electrónica: <http://revistaseug.ugr.es/index.php/sendebar/ issue/view/35/showToc>

Presas, Marisa; Pilar Cid-LeAl \& Olga TORRES-HostenCH. (2016) "Machine translation implementation among language service providers in Spain: A mixed methods study." Journal of Research Design and Statistics in Linguistics and Communication Science 3:1, pp. 126-144. Versión electrónica: <https://doi. org/10.1558/jrds.30331>

PyM, Anthony. (2003) "Redefining Translation Competence in an Electronic Age: In Defence of a Minimalist Approach." Meta, Journal des traducteurs = translators' journal 48:4, pp. 481-497. Versión electrónica: <10.7202/008533ar>

PyM, Anthony. (2013) "Translation Skills-Sets in a Machine-Translation Age." Meta, Journal des traducteurs = translators' journal 58:3, pp. 487-503. Versión electrónica: <10.7202/1025047ar>

Rico, Celia. (2017) "La formación de traductores en Traducción Automática". Revista Tradummàtica: Tecnologies de La Traducció, 15, pp. 75-96. Versión electrónica: <https://doi.org/10.5565/rev/tradumatica.200>

Rico, Celia \& Enrique TORREJÓn. (2012) "Skills and Profile of the New Role of the Translator as MT Post-editor." Revista Tradumàtica: tecnologies de la traducció 10, pp. 166-178. Versión electrónica: <https://doi.org/10.5565/rev/ tradumatica.18>

Rinsche, Adriane \& Nadia Portera-Zanotti. (2009) The size of the language industry in the EU. Studies on translation and multilingualism. Bruselas: European Commission, Directorate-General for Translation. Versión electrónica: <http://www.termcoord.eu/wp-content/uploads/2013/08/ Study_on_the_size_of_the_language_industry_in_the_EU.pdf> 
SAMSON, Richard. (2013) "El aprendizaje de las herramientas informáticas en la formación del traductor." Revista Tradumàtica: tecnologies de la traducció 11, pp. 247-256. Versión electrónica: <https://doi.org/10.5565/rev/tradumatica.54> SÁNCHEZ-GIJÓN, Pilar. (2016) "La posedición: hacia una definición competencial del perfil y una descripción multidimensional del fenómeno." Sendebar: revista de Traducción e Interpretación 27, pp. 151-162. Versión electrónica: <http:// revistaseug.ugr.es/index.php/sendebar/article/view/4016>

Schmitt, Peter A.; Lina Gerstmeyer \& Sarah Müller. (2016) CIUTI Survey 2014. Versión electronica: < https://www.researchgate.net/ publication/303495625_CIUTI-Survey2014_Schmitt>

TORRES DEL REY, Jesús. (2005) La interfaz de la traducción: formación de traductores y nuevas tecnologías. Granada: Comares.

Torres-Hostench, Olga, Pilar Cid-Leal, Pilar \& Marisa Presas (coord.). (2016) El uso de traducción automática y posedición en las empresas de servicios lingüísticos españolas: Informe de Investigación ProjecTA 2015. Bellaterra. Versión electrónica: <https://ddd.uab.cat/record/148361>

VAN DER MEER, Jaap \& Achim RUOPP. (2014) MT Market Report 2014. TAUS. Versión electrónica: <https://www.taus.net/think-tank/reports/translate-reports/ mt-market-report-2014\#summary>

VARGAS SiERRA, Chelo \& Laura RAmíREZ POLO. (2012) "The Translator's Workstation revisited: A new paradigm of translators, technology and translation." Tralogy I, Session 4 - Tools for Translators: Les Outils Du Traducteur. Versión electrónica: <http://lodel.irevues.inist.fr/tralogy/index.php?id=71>

VARIOS AUTORES (ACT). (2005) Estudio de mercado de la traducción en España: 2004. Las Rozas; Barcelona. ACT.

VARIOS AUTORES (ANECA). (2004) Libro Blanco: título de grado en traducción e interpretación. Madrid: Agencia Nacional de Evaluación de la Calidad y Acreditación. Versión electrónica: <http://www.aneca.es/var/media/150288/ libroblanco_traduc_def.pdf>

VARIOS AUTORES (EMT). (2017) Competence framework 2017. Versión electrónica: <https://ec.europa.eu/info/sites/info/files/emt_competence_fwk_2017_en_ web.pdf>

VARIOS AUTORES (EUATC). (2017) Expectations and Concerns of the European Language Industry: 2017. Versión electrónica: <https://ec.europa.eu/info/sites/ info/files/2017_language_industry_survey_report_en.pdf>

VARIOS AUTORES (ISO Technical Committees). (2017) ISO 18587: translation services: post-editing of machine translation output: requirements = Services de traduction: Post-édition d'une texte résultant d'une traduction automatique : exigences. Ginebra: ISO. 
VARIOS AUTORES (OPTIMALE). (2012) The OPTIMALE employer survey and consultation. European Commission. Versión electrónica: <https://www.euatc.org/ images/Optimale_WP4_Synthesis_report.pdf>

VARIOS AUTORES (OPTIMALE). (2013) Optimising professional translator training in a multilingual Europe: Final Report: Public part. European Commission, EACEA. Versión electrónica: <http://eacea.ec.europa.eu/LLp/project_reports/ documents/erasmus/ENW/eras_enw_177295_fr.pdf>

VARIOS AUTORES (TradOnline). (2011) Translation Business and Translators: Translation Industry Survey 2010-2011: What's new since 2008? París. Versión electrónica: <http://ec.europa.eu/translation/LID/index.cfm?fuseaction=main. PublicationContent\&PBL_ID=409\&theme_selector=normal>

Veiga DíAz, María Teresa. (2013) "La formación especializada en el nivel de posgrado en España: másteres y doctorados en traducción con componente tecnológico." Revista Tradumàtica: tecnologies de la traducció 11, p. 313-326. Versión electrónica: <https://doi.org/10.5565/rev/tradumatica.41>

ZaretSKayA, Anna; Gloria Corpas Pastor \& Míriam Seghiri Domínguez. (2015)

"Translators' Requirements for Translation Technologies: A User Survey." En: Corpas Pastor, Gloria; Míriam Seghiri Domínguez; Ruth Gutiérrez Florido \& Míriam Urbano Mendaña (eds.) 2015. AIETI7. Nuevos horizontes en los Estudios de Traducción e Interpretación. Ginebra: Tradulex, pp. 247-254. Versión electrónica: <http://www.tradulex.com/varia/AIETI7-proc.pdf>

\section{BIONOTAS / BIONOTES}

Pilar Cid-Leal. Es doctora en Ciencias de la Comunicación, licenciada en Historia y graduada en Biblioteconomía y Documentación. Profesora Titular de la Universitat Autònoma de Barcelona (UAB), donde empezó a trabajar en 1990, ha impartido numerosas materias en diversas titulaciones y facultades. Como investigadora, desde 1990 ha participado en doce proyectos de I+D financiados. Publica regularmente trabajos sobre Documentación, Traducción e Innovación docente. Es miembro del grupo Tradumàtica y codirectora de la revista digital Tradumàtica, especializada en tecnologías de la traducción. Sus líneas de investigación actuales son la Documentación aplicada a la Traducción y la Documentación general.

PILAR CID-LEAl has a PhD in Communication Science, a BA in History and a BA in Library and Information Science. She is a Senior Lecturer at the Universitat Autònoma de Barcelona (UAB), where she started working in 1990. She has taught courses in various degrees. Since 1990 he has participated in twelve subsidized R\&D projects. She regularly publishes works on Information Science, 
Translation and Teaching Innovation. She is a member of the Tradumàtica Group and the co-director of the digital journal Tradumàtica, specialized in translation technologies. Her current research lines are Information Science applied to translation and Library and Information Science.

MARÍA-CARMEN ESPÍN-GARCÍA es profesora de chino y traducción de chino en la Universitat Autònoma de Barcelona (UAB). Entre 2003 y 2014 ha sido profesora de español, inglés y francés en la Universidad Normal de Hebei (UNH). Entre 2008 y 2014 ha sido directora del Centro de Formación de Español del Instituto para el Intercambio Internacional de Cultura de la UNH. Es doctora en Teoría de la Traducción y Filología China. Ha traducido autores chinos contemporáneos. Sus principales líneas de investigación son la didáctica de la traducción, la didáctica del chino como lengua extranjera, la adquisición de la lectura en chino y las tecnologías de la traducción. Es miembro de CERAO (Centre d'Estudis i Recerca sobre Àsia Oriental) y del grupo de investigación Tradumàtica.

MARIA CARMEN ESPIN-GARCIA is a Lecturer of Chinese Language and Chinese Translation at the Universitat Autonoma de Barcelona (UAB). Prior to this, she was a Lecturer of Spanish, English and French at Hebei Normal University (2003-2014) and from 2008 she was the Director of the Spanish Training Centre of the Institute of International Culture Exchange of the Hebei Normal University. She has a PhD in Theory of Translation and in Chinese Philology. She has translated contemporary Chinese authors. Her main research interests are translation teaching methodology, Chinese as a foreign language and Chinese reading acquisition and translation technologies. She is a member of CERAO (Centre d'Estudis i Recerca sobre Àsia Oriental) and the Tradumatica Research Group.

MARISA PRESAS es profesora titular de la Universitat Autònoma de Barcelona (UAB). Imparte docencia de traducción alemán-español y metodología de la investigación. Es miembro del Grupo de Investigación PETRA (Especialización y Medio Ambiente en Traducción), miembro de la red TREC (Translation, Research, Empiricism, Cognition) y miembro del Grupo Tradumàtica. Ha publicado artículos en Target, The Interpreter and Translator Trainer y otras revistas, así como capítulos de libros en Peter Lang, Frank \& Timme, Bloomsbury y Wiley. Su investigación se centra principalmente en la didáctica de la traducción y las tecnologías de la traducción.

MARISA PRESAS is Senior Lecturer at Universitat Autònoma de Barcelona (UAB). Her teaching areas are German-Spanish Translation and Research Methodology. 
She is a member of the PETRA Research Group (Expertise and Environment in Translation, Spanish acronym), a member of the TREC network (Translation, Research, Empiricism, Cognition) and a member of the Tradumàtica Group. She has published articles in Target, The Interpreter and Translator Trainer and other journals, and book chapters in Peter Lang, Frank \& Timme, Bloomsbury, and Wiley. Her research focuses mainly on translation teaching methodology and translation technologies. 